\title{
Reconstruction of Atrial Signals Derived from the 12-lead ECG using Atrial Signal Extraction Techniques
}

\author{
P Langley ${ }^{1}$, JJ Rieta $^{2}$, M Stridh $^{3}$, J Millet-Roig $^{2}$, L Sörnmo $^{3}$, A Murray ${ }^{1}$ \\ ${ }^{1}$ Medical Physics Department, Freeman Hospital, Newcastle upon Tyne, UK \\ ${ }^{2}$ BeT-DIEo, Polytechnic University of Valencia, Spain \\ ${ }^{3}$ Department of Electroscience, Lund University, Sweden
}

\begin{abstract}
Several techniques are available for the extraction of the atrial signal from the ECG. Two of these techniques, Independent Component Analysis (ICA) and Principal Component Analysis (PCA), derive global atrial signals which cannot be directly compared with the atrial signals in specific leads. An algorithm for projecting into lead VI the global atrial signals of these techniques was developed, allowing direct comparisons with the atrial activity in VI and the atrial signal extracted by Spatiotemporal QRST cancellation (STC) which derives the atrial signal directly from the ECG lead. The reconstructed atrial signals were divided into sections corresponding to ventricular activity and inactivity in lead VI and compared against atrial activity in lead VI in terms of amplitude and waveform shape.
\end{abstract}

\section{Introduction}

We have reported previously that three techniques for the extraction of the atrial signal from the 12-lead ECG gave close agreement in measured dominant atrial frequencies [1]. To allow a direct comparison of the extracted time domain atrial signals it is necessary to apply a reverse transform to the ICA and PCA techniques. Our aim in this study was to investigate the reconstruction of the atrial signal in a specific lead for the ICA and PCA techniques. Further we compared the atrial signals extracted by each of the techniques.

In atrial fibrillation the amplitude of the atrial signal is variable, however, since the atrial and ventricular activities are decoupled, it can be assumed that the ventricular activations have no effect on the atrial signal amplitude. Therefore, the atrial signal amplitude in times of ventricular inactivity should statistically be the same as the amplitude in times of ventricular activity. This hypothosis allows the quality of the extracted atrial signals to be assessed across the full cardiac cycle.

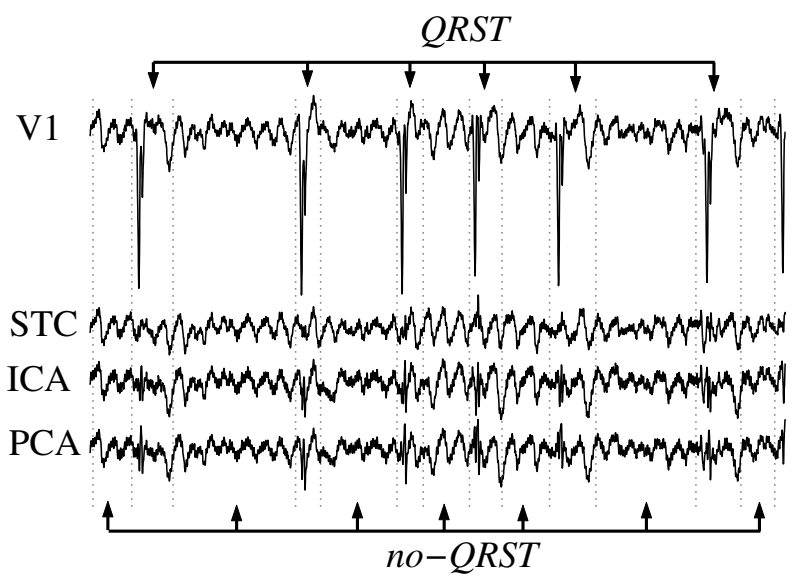

Figure 1. Illustration of $Q R S T$ and no-QRST sections.

\section{Methods}

\subsection{Data and preprocessing}

12-lead ECGs from 7 patients in atrial fibrillation recorded at the Freeman Hospital were analysed. A $10 \mathrm{~s}$ section of the ECG for each patient was analysed. Additionally a $60 \mathrm{~s}$ section was used to generate QRST templates for the STC technique. Prior to analysis, the sections of each $10 \mathrm{~s}$ recording in lead V1 containing ventricular activities (identified as QRST sections) and sections containing no ventricular activities (identified as no-QRST sections) were selected manually (figure 1).

\subsection{Extraction of the atrial signal}

The techniques developed by each of the respective research groups were used. ICA was applied by the Valencia group and PCA by the Newcastle group [2][3]. These techniques separated the atrial and ventricular activities and noise into different signals (transformed signals). The separated atrial signal or signals may have contributions from each lead and represent a global measure 
of the atrial activity. Mathematically, these techniques can be described by the linear transformation

$$
s_{i}=\sum_{j=1}^{12} A_{i, j} l(j)(i=1: 12)
$$

where, $A$ is a matrix of transform coefficients specific to the technique in question, $l(j)$ represents the $j t h$ lead from the 12-lead ECG, and $s_{i}$ are the transformed signals.

STC was applied by the Lund group. This technique derives the atrial signal directly from the ECG leads by subtraction of QRST templates derived from average beat groups from ECGs of duration $60 \mathrm{~s} \mathrm{[4].}$

\subsection{Derivation of atrial signals in lead V1 for ICA and PCA}

To allow direct comparisons between the time domain atrial signals produced by each of the techniques it was necessary to apply a reverse transform to the ICA and PCA atrial signals, effectively projecting the global atrial signals derived by these techniques back into the ECG leads. The reverse transform can be described by

$$
\hat{l}(j)=\sum_{i=k_{1}}^{k_{n}} A_{i, j}^{-1} s_{i}(k \in 1: 12)
$$

where, $\hat{l}(j)$ is the reconstructed $j^{\text {th }}$ ECG lead. The selection of transformed signals $\left(s_{i}\right)$ to include in the reverse transform is important as this affects the properties of the atrial signal. Logically these would be the ones containing only the atrial activities, but these are often distributed across several transformed signals. In order to automate this selection all possible combinations of transformed signals $\left(2^{12}-1\right.$ combinations) were used to reconstruct lead $\mathrm{V} 1$ and the set providing the optimum atrial signal was selected. The number of tranformed signals is dependent upon the characteristics of the ECG under analysis, but would typically be less than 6 . We restricted our analysis to lead V1 as this is the lead which generally exhibits most clearly the atrial fibrillation waveform, but could be applied to other leads.

Several algorithms for determining the optimal atrial signal were considered. Algorithms which compare atrial signal features in no-QRST sections alone fail since, by including all the transformed signals, both ICA and PCA would perfectly reconstruct the original signal in these sections, but at the same time they would perfectly reconstruct the QRS and T waves in the QRST sections. An algorithm which minimized the following error function was found to provide the optimum signals in the majority of cases and was used in this study:
$e=\sqrt{\left(A_{n o-Q R S T}-\hat{A}_{Q R S T}\right)^{2}+\left(A_{n o-Q R S T}-\hat{A}_{n o-Q R S T}\right)^{2}}$

$A_{n o-Q R S T}$ is the mean peak-to-peak amplitude of V1 in no-QRST sections, $\hat{A}_{Q R S T}$ and $\hat{A}_{n o-Q R S T}$ are the mean peak-to-peak amplitudes of the reconstructed lead V1 in QRST and no-QRST sections respectively. The algorithm selected the combination of transformed signals which gave the least combined error in atrial signal amplitude in both QRST and no-QRST sections.

\subsection{Comparing the atrial signals}

The aim of atrial signal extraction is to remove QRS and $\mathrm{T}$ waves without distorting the atrial component of the ECG. Quantifying the quality of the extracted atrial signals is difficult since the true atrial signal on the body surface during the QRS complex and T wave is unknown. It was expected that the atrial activity during ventricular activity would be similar to that during ventricular inactivity, since the ventricular and atrial activities in atrial fibrillation are decoupled. With this assumption it was possible to compare statistically the amplitudes of the atrial signals within and without periods of ventricular activity. This was achieved by measuring the mean and standard deviation of peakto-peak amplitude across no-QRST sections of $\mathrm{V} 1$, and similarly, the peak-to-peak amplitude of the reconstructed atrial signals in $Q R S T$ sections. The mean amplitudes were compared using the t-test.

It was also anticipated that the extracted and original atrial signals, measurable during periods of ventricular inactivity, would be morphologically the same, excepting distortions produced by the extraction technique. Therefore, to quantify morphological distortions of the atrial signal due to the extraction (and reconstruction) process, we compared the amplitudes of the extracted atrial signals in no-QRST sections with those of $\mathrm{V} 1$ in the same sections using the paired t-test. Additionally, the mean square errors (MSE) between original and extracted signals of these sections were calculated.

\section{Results}

Figure 2 compares the amplitudes of the atrial signal in no-QRST sections of $\mathrm{V} 1$ to the extracted atrial signals in both no-QRST sections and QRST sections. Significant differences in amplitude are indicted. Table 1 summarises the results from this figure by quantifying for each technique the number of significant increases or decreases in atrial signal amplitude compared to that of V1.

MSE for each of the techniques is shown in figure 3. 


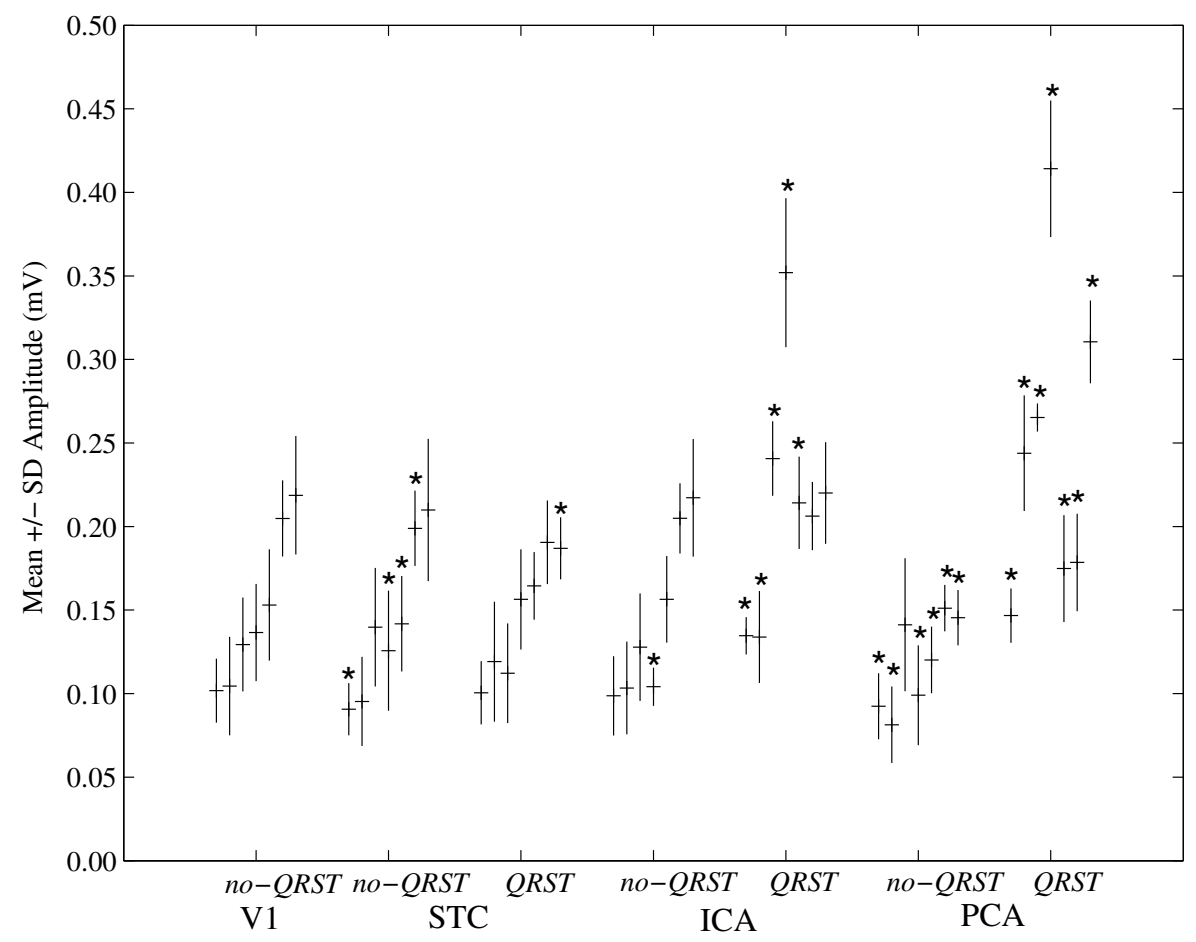

Figure 2. Atrial signal amplitudes of $Q R S T$ and no-QRST sections for all patients (in order 1 to 7 ) for STC, ICA and PCA compared with amplitudes in V1. * indicates $\mathrm{p}<0.05$ for amplitudes compared with V1 no-QRST.

Table 1. The number of patients for which there were significant increases $(\uparrow)$ or decreases $(\downarrow)$ in extracted atrial signal amplitudes in no-QRST and QRST sections compared with no-QRST sections in V1.

\begin{tabular}{llllll}
\hline & \multicolumn{2}{c}{$n o-Q R S T$} & & \multicolumn{2}{c}{$Q R S T$} \\
\cline { 2 - 3 } \cline { 5 - 6 } & $\uparrow$ & $\downarrow$ & & $\downarrow$ \\
\hline STC & 0 & 4 & & 0 & 1 \\
ICA & 0 & 1 & & 5 & 0 \\
PCA & 0 & 6 & & 7 & 0 \\
\hline
\end{tabular}

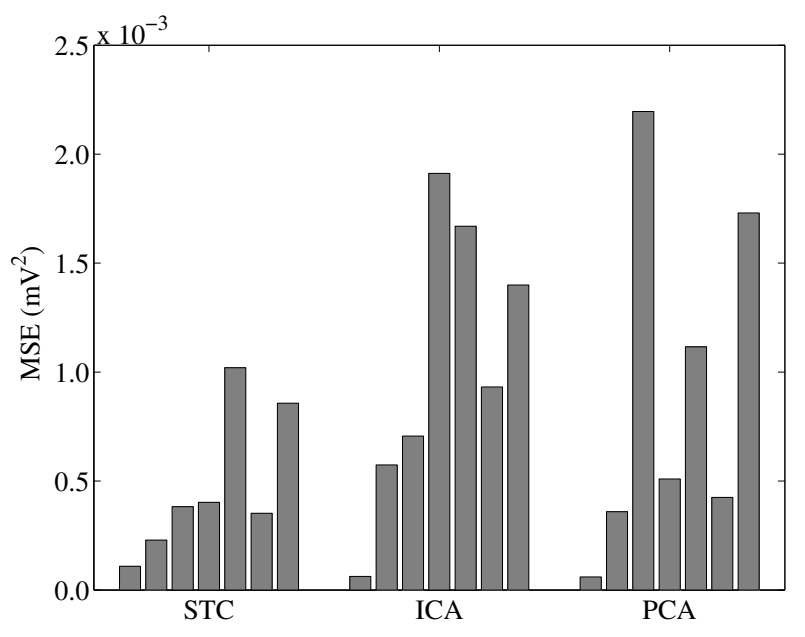

Figure 3. MSE for all patients (in order 1 to 7 ) by each technique. 

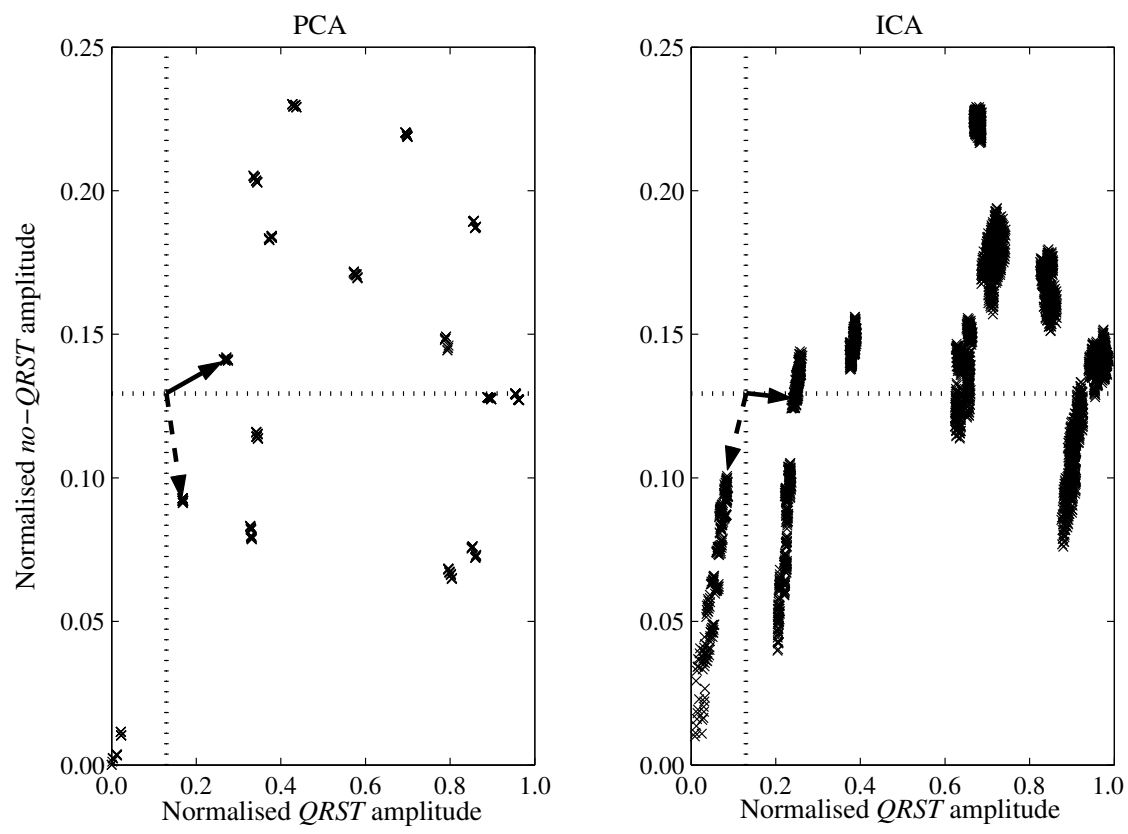

Figure 4. Normalised QRST and no-QRST amplitudes for all reconstructed V1 leads for patient 3 for PCA and ICA. Dashed lines indicate target atrial signal amplitude. Solid arrow indicates the reconstruction selected by the algorithm. Dashed arrow indicates possible alternative reconstruction but which results in reduced atrial signal amplitude.

\section{Discussion}

This is the first study to reconstruct the atrial activity in V1 from the extracted atrial signals of the ICA and PCA techniques. One of the problems has been determining which set of transformed signals to use in the reconstruction to give the optimal atrial signal. Figure 4 relates the amplitude of the $Q R S T$ and no-QRST sections in $2^{12}-1$ reconstructed V1 leads for ICA and PCA techniques for patient 3. The algorithm used in this study selects the reconstruction which minimises the distance from the origin ( $\left.x=y=A_{n o-Q R S T}\right)$, as indicated by the solid arrow in figure 4. In this example, for both ICA and PCA techniques, the selected atrial signals have amplitudes in the $Q R S T$ section that are approximately twice that of the atrial amplitude in V1. Selection of other reconstructions for example, as indicated by the dashed arrow in figure 4, would bring the amplitude of the QRST section closer to the target value but at the cost of reduced amplitude in the no-QRST section. It is apparent that in this case no reconstruction is possible which would give good agreement in atrial signal amplitude in both QRST and noQRST sections. The STC technique has the advantage that it derives the atrial signal directly from the lead in question and this technique may be better for analysis of specific leads of the 12-lead ECG, whereas the other techniques may be better at providing global information relating to atrial activities.

\section{Acknowledgements}

P Langley is supported by the Engineering and Physical Sciences Research Council.

\section{References}

[1] Langley P, Stridh M, Rieta JJ, Sörnmo L, Millet-Roig J, Murray A. Comparison of atrial rhythm extraction techniques for the estimation of the main atrial frequency from the 12lead electrocardiogram in atrial fibrillation. Computers in Cardiology 2002;29:29-32.

[2] Rieta JJ, Zarzoso V, Millet-Riog J, Carcía-Civera R, RuizGranell R. Atrial activity extraction based on blind source separation as an alternative to QRST cancellation for atrial fibrillation analysis. Computers in Cardiology 2000;27:6972.

[3] Langley P, Bourke JP, Murray A. Frequency analysis of atrial fibrillation. Computers in Cardiology 2000;27:65-68.

[4] Stridh M, Sörnmo L. Spatiotemporal QRST cancellation techniques for analysis of atrial fibrillation. IEEE Trans Biomed Eng 2001;48:105-111.

Address for correspondence:

Philip Langley

Medical Physics Department, Freeman Hospital

Newcastle upon Tyne, NE7 7DN, UK.

philip.langley@ncl.ac.uk 